Ivo J. T. M. Claassen ${ }^{1}$, Ab D. M. E. Osterhaus ${ }^{2}$ and Eric Claassen $^{3,4}$

${ }^{1}$ Laboratory for Control of biological products, National Institute for Public Health and Environmental Protection, Bilthoven, The Netherlands

${ }^{2}$ Department of Virology, Erasmus University, Rotterdam, The Netherlands

${ }^{3}$ Division Immunological and Infectious Diseases, TNO Prevention and Health, Leiden, The Netherlands

${ }^{4}$ Department of Immunology, Erasmus University, Rotterdam, The Netherlands

\section{Antigen detection in vivo after immunization with different presentation forms of rabies virus antigen: involvement of marginal metallophilic macrophages in the uptake of immune-stimulating complexes}

\begin{abstract}
Several mechanisms have been postulated to explain the relatively high immunogenicity of antigens presented in immune-stimulating complexes (iscom). Their potency can in part be explained by the specific targeting of these structures to cells presenting antigens to the immune system. However, until now no method for the subcellular detection of iscom in situ was available. In the present study, a novel, fast and simple method for the detection of iscoms in situ is demonstrated. By making use of the lipophilic fluorescent carbocyanine dyes, 1, $1^{\prime}$-dioctadecyl-3,3,3',3'-tetramethylindocarbocyanine perchlorate (DiI) and $3,3^{\prime}$-dioctadecyloxacarbocyanine perchlorate $(\mathrm{DiO})$, rabies virus antigen and iscom prepared with this antigen were visualized with fluorescence microscopy. The labeled antigen and iscoms were observed in macrophages of spleen and liver of mice within 1-2 h after intravenous administration. When administered intramuscularly or in the footpad, uptake in macrophages of draining lymph nodes could be demonstrated. In the spleen, labeled inactivated virus antigen localized preferentially in the marginal zone macrophages and to a lesser extent in the red pulp macrophages. In contrast, antigen presented in iscom was taken up mainly by the marginal metallophilic macrophages and to a much lesser extend by marginal zone macrophages or follicular-dendritic and -B cells. This method enables the detection of iscom and membrane viruses and allows the analysis of their relation to antigen-presenting cells in situ. Here, we demonstrate that iscom containing rabies virus antigen are taken up by a subset of macrophages in the spleen distinct from those that take up inactivated rabies virus antigen not presented in iscom, thereby possibly explaining the observed difference in immunogenicity of these antigen preparations. Furthermore, we show a lower efficiency on the induction of humoral and cellular responses after intravenous immunization for both types of antigen when compared with subcutaneous immunization.
\end{abstract}

\section{Introduction}

Since immune-stimulating complexes (iscom) were initially described by Morein et al. [1], numerous studies have demonstrated their potential as a vaccine with built-in adjuvant properties. Bacterial, parasitic, viral and other proteins and peptides have been incorporated into iscom to enhance the immune response against these antigens [2]. Immunization with iscom generally induces both humoral

[I 13939]

Correspondence: Ivo. J. T. M. Claassen, Laboratory for Control of Biological Products, National Institute for Public Health and Environmental Protection, POB 1, 3720 BA Bilthoven, The Netherlands (Fax: + 3130293616)

\begin{abstract}
Abbreviations: Dil: 1,1'-Dioctadecyl-3,3,3',3'-tetramethylindocarbocyanine perchlorate DiO: 3,3'-Dioctadecyloxacarbocyanine perchlorate iscom: Immune-stimulating complex MZM: Marginal zone macrophages MMM: Marginal metallophilic macrophages NSE: Nonspecific esterase RPM: Red pulp macrophages RV: Rabies virus RV-BPL: $\beta$-Propiolacton-inactivated rabies virus
\end{abstract}

Key words: Immune-stimulating complex / Marginal zone / Macrophages / Fluorescence and cell-mediated immune responses [3]. The antibody responses against antigens incorporated in iscom are generally higher and longer-lasting than those induced by the same amount of antigen not incorporated in this matrix. Furthermore, unlike most inactivated antigens, iscom can induce an MHC class-I-restricted cytotoxic T cell response specific for the immunizing antigen [4-6]. Little is known about the early events after immunization with iscom, e.g. which antigen-presenting cells are involved or in which anatomical location (organ or compartment) the response is elicited. Watson et al. [7] described the inflammatory response and organ distribution of antigen after intraperitoneal immunization with influenza virus glycoprotein in iscom or micelles using radioactively labeled antigens, and showed that the spleen is important in the uptake and retention of iscom. However, no detailed information on the involvement of splenic antigen-presenting cells (e.g. macrophages, dendritic cells, B cells) in the uptake, processing and presentation of iscom or inactivated virus is available. Such knowledge would allow the study of distinct subsets of antigen-presenting cells in the differential immunogenicity of viral antigens presented in different ways. Here, we present a simple post-formation method for labeling virus and iscom by means of lipophilic fluorescent dyes. This method allows the study of virus and iscom distribution in the lymphoid organs at the cellular level. 


\section{Materials and methods}

\subsection{Animals and chemicals}

Female BALB/c mice aged 12-16 weeks, were kept in Macrolon cages under an 11-h dark/13-h light regimen at $20^{\circ} \mathrm{C}$ and were given acidified water ( $\mathrm{pH} 3$ ) and pelleted mouse food (Hope Farms, Woerden, The Netherlands) ad libitum. DiI (1,1'-dioctadecyl-3,3,3',3'-tetramethylindocarbocyanine perchlorate; D-282), and DiO (3,3'-dioctadecyloxacarbocyanine perchlorate; D-275) were obtained from Molecular Probes (Eugene, OR). Monoclonal antibodies ERTR-9, specific for marginal zone macrophages, and MOMA-1, specific for marginal metallophillic macrophages, were a kind gift of Prof. Dr. G. Kraal, Free University, Amsterdam, The Netherlands.

\subsection{Antigens}

Rabies virus (RV-Pasteur strain) was propagated in Vero cell monolayer cultures [8]. Culture supernatant was cleared by filtration and concentrated by Amicon ultrafiltration (cut-off $10^{6}$ Dalton). Virus was inactivated with B-propiolactone (BPL) and stored at $-70^{\circ} \mathrm{C}$ at a concentration of $900 \mu \mathrm{g} / \mathrm{ml}$. This rabies virus antigen was used for the preparation of RV-iscom as described [9]. Briefly, RV proteins were solubilized with $10 \%$ MEGA-10 (decanoyl$\mathrm{N}$-methylglucamide) for $2 \mathrm{~h}$ at room temperature. The solubilized virus was layered on top of a $10 \%$ sucrose cushion and centrifuged for $2 \mathrm{~h}$ at $200000 \times \mathrm{g}$. Quil-A (Iscotec AB, Lulea, Sweden), cholesterol and phosphatidylcholine (Sigma, St. Louis, MO) were added to the supernatant and the mixture dialyzed overnight against PBS. Free Quil-A and unincorporated proteins were removed by centrifugation over a $20 \%$ sucrose cushion for $48 \mathrm{~h}$ at $150000 \times \mathrm{g}$. Incorporation of $\mathrm{RV}$ proteins was analyzed by SDS-PAGE and a glycoprotein-specific ELISA $[10]$.

\subsection{Immunological responses and protection}

Immunological responses were measured 4 weeks after i.v. or s.c. immunization. RV-specific neutralizing antibody responses were measured by rapid fluorescence focus inhibition test (RFFIT) and T cell responses were measured as described $[11,12]$. Briefly, spleen cells were isolated and stimulated with different concentrations of RV-BPL. After 5 days, cells were pulsed with $\left[{ }^{3} \mathbf{H}\right]$ thymidine for $18 \mathrm{~h}$. Cells were harvested on glass filters and incorporated radioactivity was measured in a Betaplate scintillation counter (LKB, Uppsala, Sweden). For protection studies, $\mathrm{BALB} / \mathrm{c}$ mice were challenged intracerebrally with $30 \mathrm{LD}_{50}$ rabies challenge virus standard CVS-26 according to standard NIH potency testing of vaccines [13].

\subsection{Fluorochrome labeling of $\mathrm{RV}$ antigen and $\mathrm{RV}$-iscom}

Stock solutions of DiI and DiO were made in pure ethanol or DMSO $(2.5 \mathrm{mg} / \mathrm{ml})$. RV-BPL $(100 \mu \mathrm{g})$, RV-iscom or empty iscom in $1 \mathrm{ml}$ PBS were vigourously mixed with $8 \mu \mathrm{l}$ $(20 \mu \mathrm{g})$ DiI stock solution and incubated for $1 \mathrm{~h}$ at $37^{\circ} \mathrm{C}$. Any unincorporated DiI formed crystals during this incu- bation period. After labeling, the samples were filtered using $0.45-\mu \mathrm{m}$ filters to separate crystalline DiI and labeled antigens [14]. Labeled material was stored in the dark at $4^{\circ} \mathrm{C}$ for up to 4 weeks.

Total amounts of carbocyanine label incorporated in virus and iscoms were determined with a Beckman DU-40 UV/Vis spectrophotometer. Wavelength scans were made from 380 to $600 \mathrm{~nm}$ with peaks at $552 \mathrm{~nm}$ for DiI and $492 \mathrm{~nm}$ for DiO. Association of carbocyanine dye with the iscom structure was demonstrated by purification of $\mathrm{DiO}$ labeled iscom by density centrifugation on a $10-60 \%$ sucrose gradient for $18 \mathrm{~h}$ at $40000 \times \mathrm{g} \mathrm{[15]}$.

\subsection{Fluorescence microscopy}

Frozen 8 - $\mu \mathrm{m}$ sections of organs were examined and photographed directly after cryo-sectioning with an Olympus (Tokyo) VANOX fluorescence microscope. DiI fluorescence was observed both as red with green light (excitation filter BP 545) and rhodamine/TRITC optics (emission: $\mathrm{EO} 570$ ) and as golden-yellow with blue light (BP $490+$ EY 455) and FITC optics (EO 515). DiO fluorescence was observed only with FITC optics as green (excitation: BP 490 + EY 455, emission: EO 515).

\subsection{Enzyme- and immunocytochemistry}

Organ sections used for localization studies based on fluorescence microscopy were air-dried and fixed in $1 \%$ paraformaldehyde at $37^{\circ} \mathrm{C}$ for $15 \mathrm{~min}$ for simultaneous fluorescence and immunochemical detection (acetone dissolves lipid membranes, causing a significant decrease in fluorescence of labeled iscom/virus). Sections for enzyme staining (nonspecific esterase; NSE) were fixed in acetone after fluorescence observation and photomicrography. Monoclonal antibody staining on fixed sections for marginal zone metallophilic macrophages (MOMA-1) was performed as described [16]. Endogenous acid phosphatase staining for the identification of all splenic and liver phagocytes and NSE staining for the detection of marginal metallophilic macrophages (MMM) was performed as described [16]. Localization of the respective subsets of splenic macrophages has also been described [17].

\section{Results}

\subsection{Incorporation of dyes in virus and iscom}

The efficiency of the labeling of viral antigen and iscom is shown in Fig. 1. At the optimal dye concentration used (200 $\mu \mathrm{g} \mathrm{dye} / \mathrm{mg}$ iscom), up to $80 \%$ was integrated in the $\mathrm{RV}$-iscom. Under the same conditions, RV-BPL took up approximately $50 \%$ of the dye. The carbocyanine dye associates specifically with the iscom structure, as shown by sucrose gradient density centrifugation of DiO-labeled iscom and in a recent report dealing exclusively with mucosal localization of iscom [15]. To detcrmine whether the incorporation of these dyes influences the structure and immunological properties of iscom or viral antigens, labeled antigens were analyzed morphologically by electron microscopy as well as for their immunogenicity in mice. 


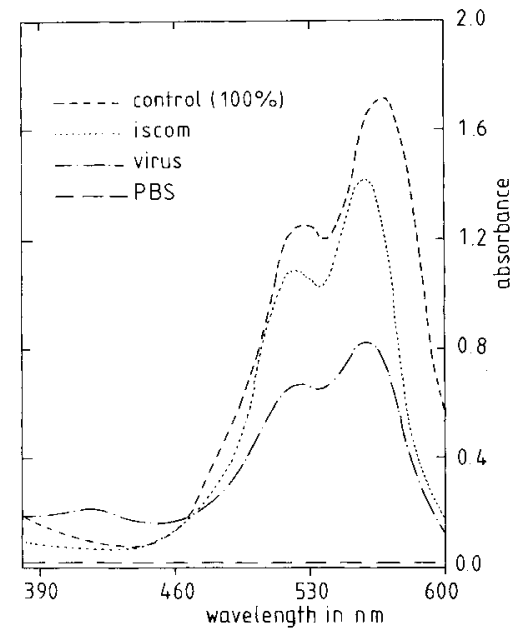

Figure 1. Incorporation of carbocyanine DiI in virus and iscom as determined by spectrophotometric measurements (wavelength scans from $390-600 \mathrm{~nm}$ ) after $2 \mathrm{~h}$ incubation (see Sect. 2.4). Control $100 \%$ contains the same amount of label as was added to the antigen preparations without filtration. Iscom, RV-BPL and PBS were all filtered to remove dye which was not take up in the lipid phasc after incubation.
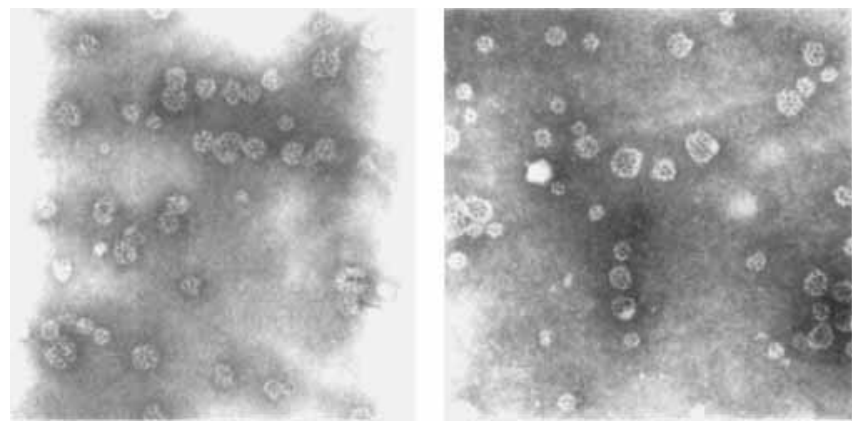

Figure 2. Electron micrographs $(\times 106000)$ of unlabeled (left panel) and DiI-labeled (right panel) iscom matrix after $0.2-\mu \mathrm{m}$ filtration: note no differences in typical cage-like iscom structure.

Fig. 2 shows representative electron micrographs of iscom matrix before (left panel) and after (right panel) DiI incorporation. No changes in the structure were observed after DiI uptake, and all samples were devoid of any particulate material other than the iscom. Results obtained with RV-BPL (data not shown) were essentially the same as for RV-iscom. Labeled virus and iscoms were shown to retain their normal immunogenic properties (data not shown) as the same results were obtained as presented in Fig. 7 for non-labeled material.

\subsection{Localization of antigen and iscom in the spleen after i.p. or i.v. injection}

After i.p. and i.v. injection, both antigens could be demonstrated in the Kupffer cells of the liver within $15 \mathrm{~min}$, shown, for iscom only, in Fig. 3a. No differences in liver localization pattern were observed between RV-BPL or RV-iscom.
When DiI-labeled antigens were injected i.p. or i.v., uptake in splenic macrophages could be observed as early as $20 \mathrm{~min}$ after administration. Labeled RV-BPL preferentially localized in marginal zone macrophages (MZM) (Fig. 3b). When large amounts of RV-BPL were administered, antigen was also taken up by red pulp macrophages (RPM). Viral antigen was detected in roughly equal concentration ratios in MZM and RPM after 1-7 days irrespective of the dosage given.

Labeled iscom were taken up by MZM as shown for other antigens. However, iscoms preferentially localized within MMM. As shown in Fig. 4, a section showing the uptake of DiI-labeled iscoms in the spleen was photographed (Fig. 4a) and subsequently fixed in acetone. Following fixation, the section was incubated with a substrate for NSE, an MMM endogenous enzyme marker (Fig. 4b). It is clear from these pictures that similar patterns are obtained for Dil-labeled iscoms and NSE activity. Although iscoms were not found exclusively in MMM, but also in MZM, almost all NSE-containing macrophages can be seen to have ingested iscoms, whereas RV-BPL could not be observed in these cells.

The involvement of MMM in the uptake of iscom was also demonstrated by a double-fluorescence study. First, DiIlabeled iscom were injected in mice, and after $2 \mathrm{~h}$ the spleen was removed, frozen, cryo-sectioned and paraformaldehyde-fixed. After fixation, sections were incubated with MOMA-1 antibody and goat anti-rat conjugated to FITC. Using FITC optics, DiI iscoms were observed as golden yellow and MOMA-1-staining cells as green. FITC did not fluoresce when using green light for excitation, showing iscom in red. Most MOMA-1 - positive cells were positive for DiI fluorescence, albeit with differences in intensity, and hence for iscom uptake (data not shown).

Follicular localization (either on follicular-dendritic or -B cells) of iscom was also observed [green dots in F (follicle) Fig. 5] in a similar fashion as that shown for TNP-Ficoll [18] and for liposomes [19].

\subsection{Double-fluorescence labeling studies}

To confirm the differences in localization pattern of RVBPL and RV-iscom, the following experiment was performed. RV-BPL was labeled with DiI and RV iscom with DiO. When observed using FITC optics, these labels fluoresce golden yellow for RV-BPL and green yellow for iscom, respectively. Mixtures of these antigens were injected intravenously, and after $2 \mathrm{~h}$, the spleens were removed. Observation of sections revealed that RV-BPL and iscom co-localized in MZM, but a small ring of cells on the border between marginal zone and periarteriolar lymphocyte sheath (PALS) contained only iscom (green yellow) and no labeled virus material (Fig. 5).

\subsection{Localization after intramuscular or subcutaneous injection}

When RV-BPL or RV-iscom were injected s.c. or i.m., both antigens were rapidly found in the draining lymph nodes. 


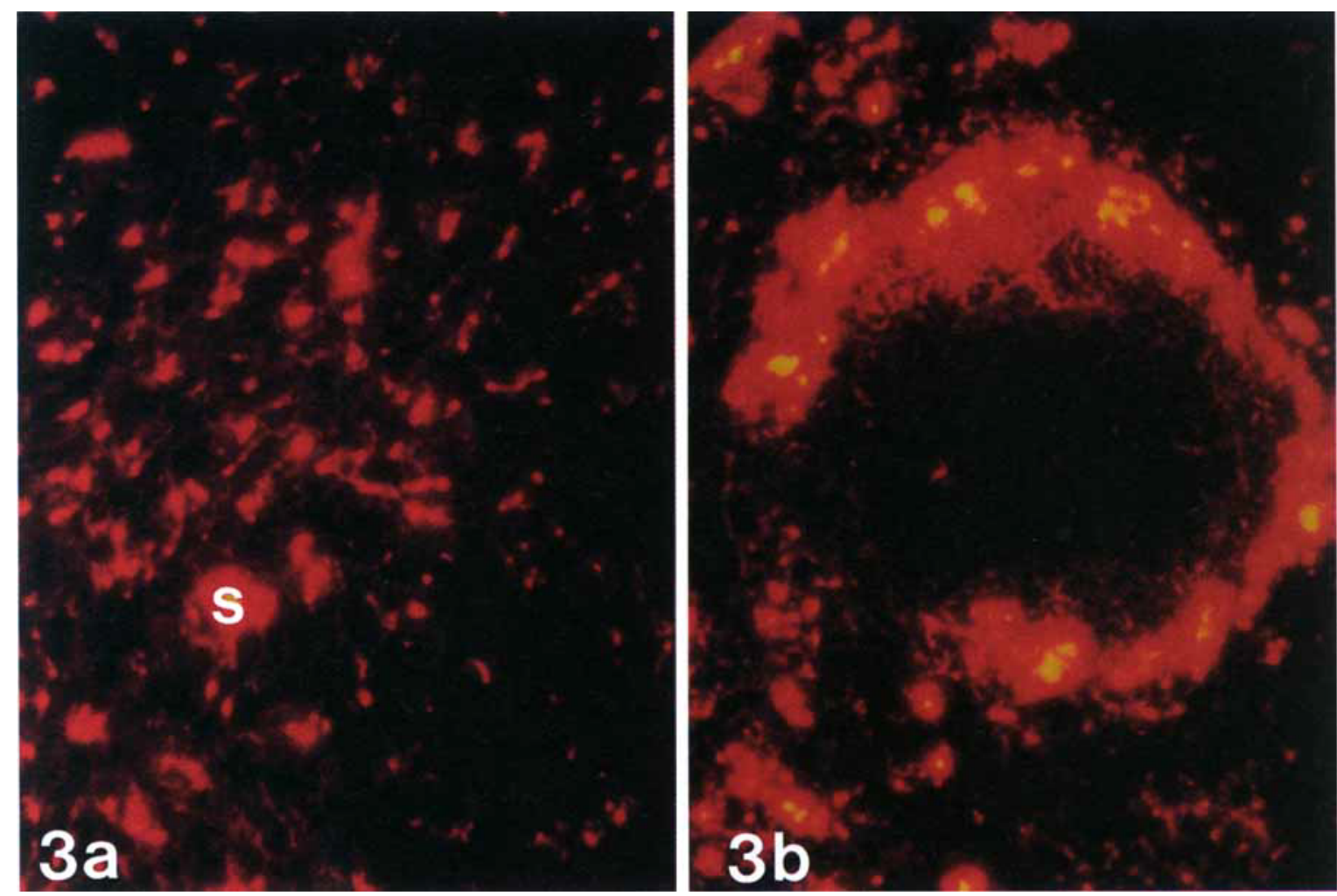

Figure 3. (a) Fluorescence microscopic image of an 8 - $\mu \mathrm{m}$ liver cryo-section showing DiI-iscom localization in Kuppfer cells $2 \mathrm{~h}$ after intraperitoneal injection. $\mathrm{S}=$ sinus. (b) Fluorescence microscopic image of an 8 - $\mu \mathrm{m}$ spleen cryo-section showing localization of RV in a typical ring of $\mathrm{MZM}, 2 \mathrm{~h}$ after i.v. injection.

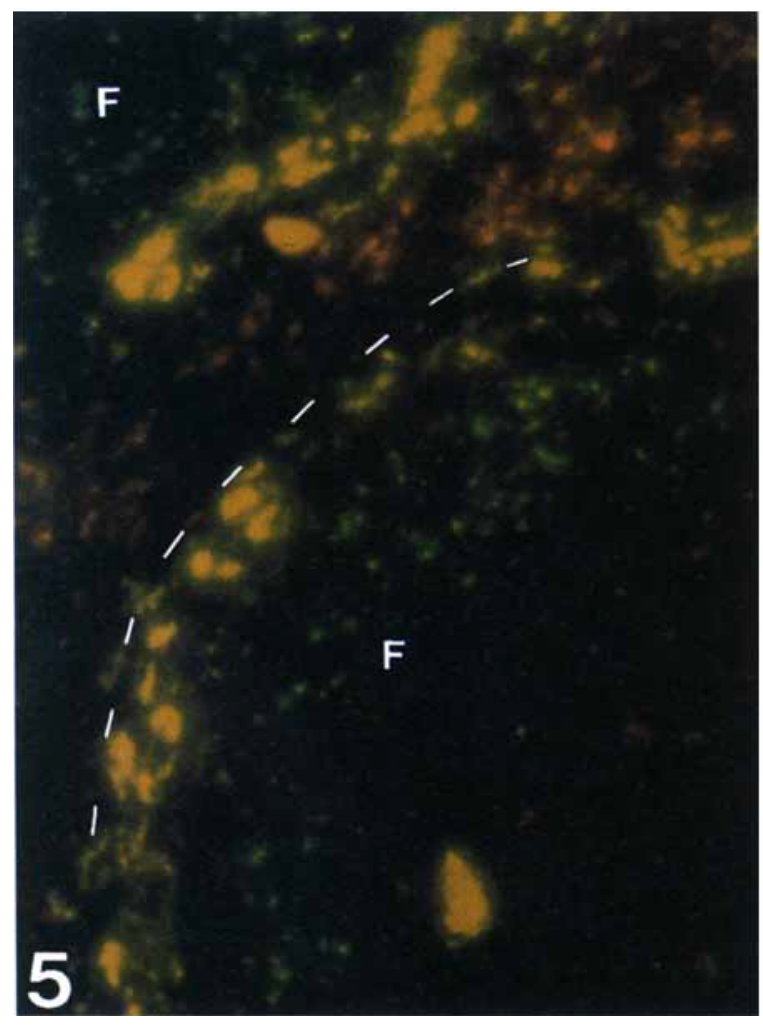

Figure 5. This shows the different localization of DiI-labeled rabies virus (golden yellow) in MZM and DiO-labeled iscoms (green yellow) after i.v. injection. Two follicles (F) are segregated by the marginal zone. MMM are located on the border. The dashed line indicates the border between MMM and MZM. Also, follicular localization of the DiO-labeled iscoms (green spots) in the follicle can be seen.

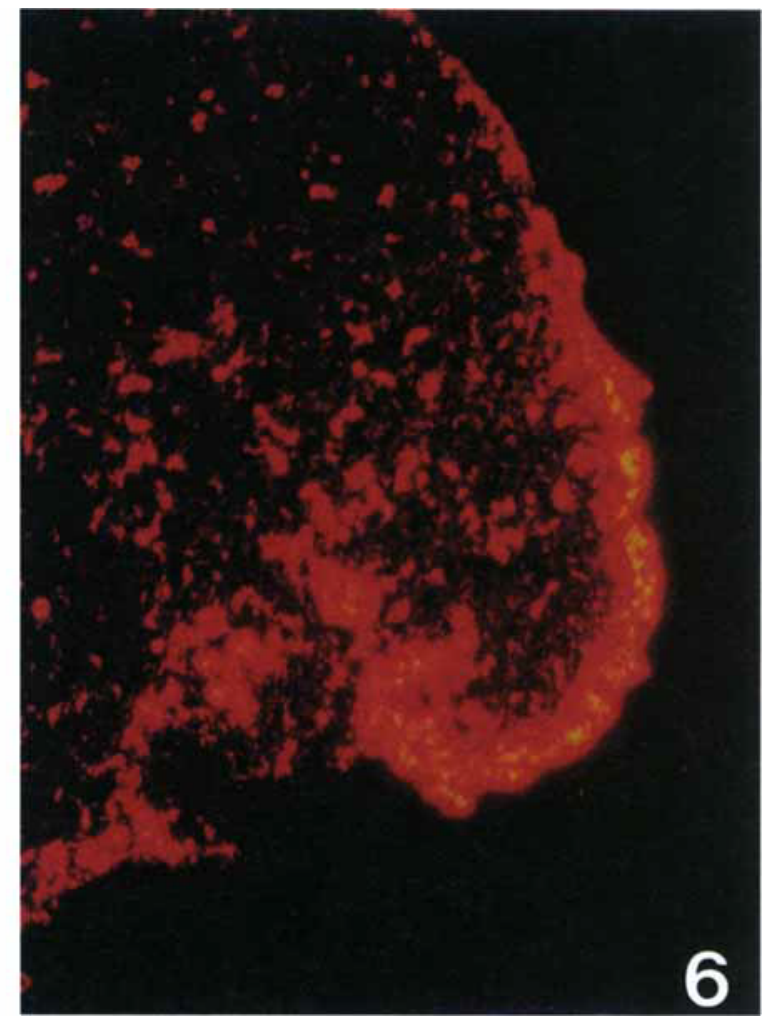

Figure 6. Fluorescence microscopic image of an 8-um cryo-section showing Dil-iscom localization in the popliteal lymph node $2 \mathrm{~h}$ after s.c. injection in the footpad. 
Intramuscular injection in the hind leg resulted in the uptake of both antigens in popliteal and inguinal lymph nodes. Footpad-injected antigens were found in the popliteal lymph node only. In lymph nodes, labeled antigens localized initially in the subcapsular macrophages, and after a few hours in the medullary macrophages. Localization is shown for s.c.-injected iscom only in Fig. 6. No differences between RV-BPL or RV-iscom localization patterns or intensity were observed.
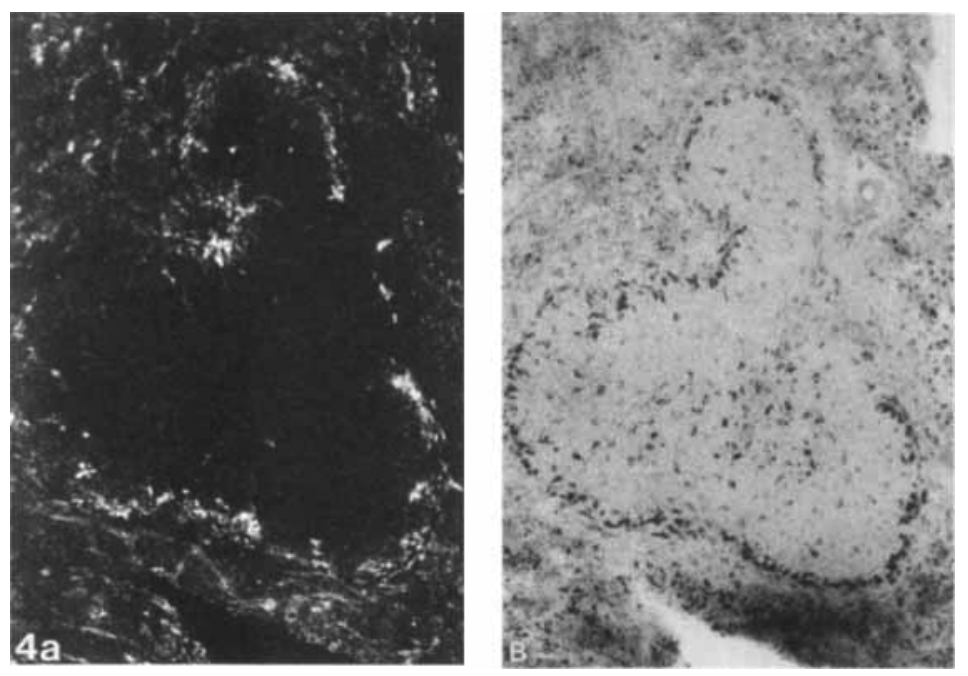

Figure 4. (a) Fluorescence microscopic image of an $8-\mu \mathrm{m}$ spleen cryo-section showing Dil-iscom loaclization $2 \mathrm{~h}$ after i.v. injection. (b) Shows the same section after acetone fixation and incubation with NSE substrate, specifically enzymatically staining the MMM.
RABIES-SPEGIFIC VIRUS NEUTRALIZING Ab

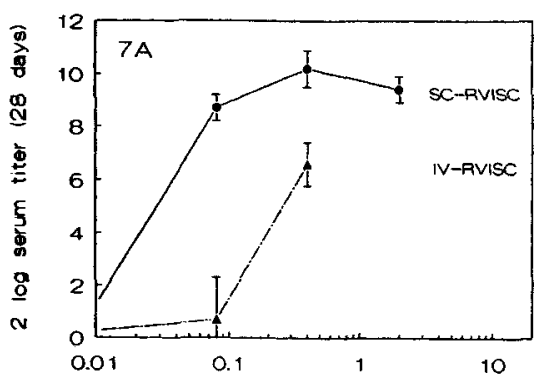

重

RABIES SPECIFIC CD4 T CELL PROLIFERATION

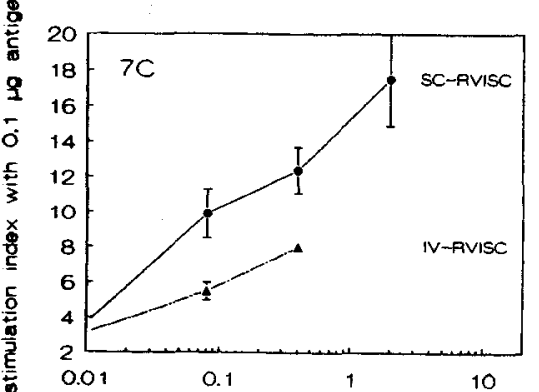

SLRVIVAL AFTER CHALLENGE (LIVE VIRUS)
RABIES-SPECIFIC VIRUS NEUTRALIZING Ab

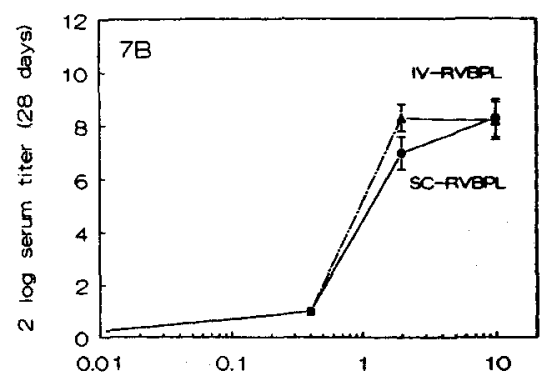

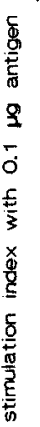

RABIES SPECIFIC CO4 T. CELL PROLIFERATION

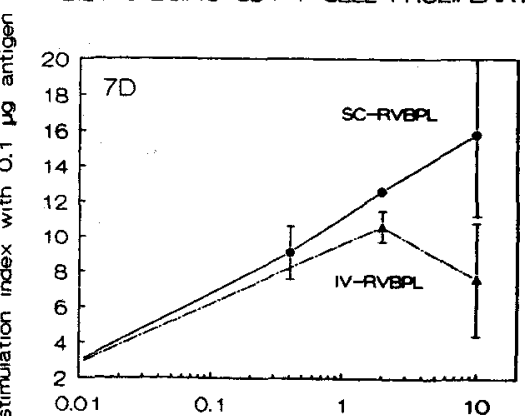

SURVIVAL AFTER CHALIENGE (LIVE VIRUS)

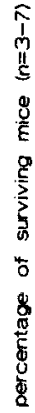

antigen dose (microgram) per mouse

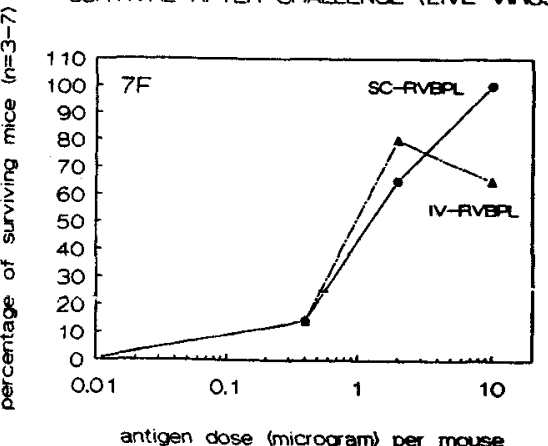

Figure 7. (A) and (B) show the serum titer of RV-specific neutralizing antibodies 28 days after a single immunization with different antigens. (C) and (D) show $\mathrm{RV}$-specific $\mathrm{T}$ cell responses of immune spleen cells when stimulated with $0.1 \mu \mathrm{g}$ inactivated rabies virus in vitro. (E) and (F) show the percentage of survival following challenge with $30 \mathrm{LD}_{50}$ live CVS21 after a single immunization with different antigens. Virus neutralizing titers and $T$ cell stimulation indices are mean values for at least three animals in a representative experiment. Survival data after challenge were determined in separate experiments. SC-RVBPL: BPL-inactivated RV given subcutaneously, SC-RVISC: RV iscom given s.c., SHAM: no immunization, IV-RVBPL: BPL-inactivated RV given i.v., IV-RVISC: RV iscom given i.v. 


\subsection{Immunological responses after immunization with RV-BPL and RV-iscom}

In view of the observed differences in localization patterns of RV-BPL and RV-iscom after i.v. and i.p. injection, mice were immunized to study the induction of $\mathrm{B}$ and $\mathrm{T}$ cell responses and protection against challenge with virulent $\mathrm{RV}$. The results, of which a representative experiment is shown in Fig. 7 , showed that in mice, RV-iscom are about 20-30-fold more potent than RV-BPL when injected subcutanously. RV-iscom and RV-BPL both induced good virus-neutralizing antibodies via both routes (Fig. 7A,B). Via the s.c. route, $0.08 \mu \mathrm{g} \mathrm{RV}$-iscom induced $\mathrm{RV}$-neutralizing antibody titers as high as those found with $10 \mu \mathrm{g}$ RV-BPL, demonstrating the higher immunogenicity of the RV-iscom. When administered i.v., comparable amounts of $\mathrm{RV}$-iscom induced significantly lower titers. T cell proliferation in vitro after stimulation with RV-BPL was used as a measure of $\mathrm{T}$ cell priming in vivo. Fig. 7C,D show that $\mathrm{RV}$-iscom prime $\mathrm{T}$ cells more efficiently and at lower doses than RV-BPL. Immunization doses as low as $0.08 \mu \mathrm{g}$ $\mathrm{RV}$-iscom still elicited $\mathrm{T}$ cell responses in vitro, and these data corresponded with the induction of neutralizing antibody titers.

After intracerebral challenge with $30 \mathrm{LD}_{50}$ CVS-26, all sham-treated animals $(n=7)$ died (data not shown). Full protection was obtained with iscom with a dose as low as $0.4 \mu \mathrm{g}(n=6)$ when given via the subcutaneous route (Fig. 7E). RV-BPL was fully protective at a dose of $10 \mu \mathrm{g}$ s.c. (Fig. 7F). When given intravenously, RV iscom were two- to fivefold less effective, but full protection could still be obtained with $2 \mu \mathrm{g} \mathrm{RV}$-iscom i.v. $(n=3)$. Taken together, these data confirm the stronger immunogenicity of proteins incorporated into iscom, and also show a lower efficiency of the i.v. route of immunization over the s.c. route, especially for $\mathrm{RV}$-iscom.

\section{Discussion}

$\mathrm{RV}$-iscom, in contrast to inactivated $\mathrm{RV}$ antigen, are preferentially taken up by MMM of the murine spleen after i.v. or i.p. administration. This is an interesting finding since, until now, no involvement of this particular subset of macrophages in antigen uptake has been demonstrated. $\mathrm{RV}$-iscom were more immunogenic than RV-BPL in terms of induction of virus-neutralizing antibodies, $T$ cell responses, and protection against disease. The subcutaneous immunization route, which primarily activates the draining lymph nodes, proved more efficient than immunization via the i.v. route. Furthermore, it was shown that carbocyanine dyes can be used effectively to label membrane viruses and iscom, permitting localization pattern studies of these antigens in situ.

DiI and DiO were taken up within a few seconds in viral membranes and iscom. The iscom matrix is highly hydrophobic and membrane proteins are easily incorporated during its formation. Due to this property and the lipophilic character of DiI and DiO, these dyes are easily incorporated in the iscom structure. It should be stressed that these carbocyanine dyes are taken up exclusively in lipophilic structures, i.e. membranes, and, therefore, do not bind to or interfere with function or immunogenicity of surface proteins [14]. The use of antigens labeled in this way has enabled us to obtain data on the early processes of antigen handling in vivo.

Recently, this method was used to study the kinetics of liposome uptake in which MZM and RPM play an important role [14]. In this study, it was surprising to find that there are indeed differences in the cell populations which are involved in uptake of the antigens when they enter the spleen. MZM are known to play an important role in the uptake of particulate antigens (e.g. liposomes, bacteria) since they are among the first cells encountered upon entry in the spleen.

Labeled RV and RV-iscom could be found in MZM within $2 \mathrm{~h}$ after i.v. or i.p. injection, as would be expected. However, only iscom were found in MMM, a cell population which is located at the border of the marginal zone, characterized by slender processes protruding into the outer PALS, which is the main site of antibody production in the spleen. The role of these cells in the handling of antigens is unclear [20] but their involvement in the processing of antigens has been suggested by Kraal et al. $[16]$.

It should be noted that studies recently showed that selective uptake and retention of antigens by the MZM in vivo functions as a way of rapid antigen removal and elimination, thereby obscuring it from the immune system and hampering a normal response [21]. This may explain the relative inefficiency of RV-BPL taken up by MZM to induce immune responses at low doses, in contrast to results found with iscom, which are taken up by MMM. Similarly, the fact that significant amounts of both antigens given by the i.v. or i.p. route are taken up by the MZM could also explain the relative inefficiency of this route.

The importance of the microenvironment for antigen processing and presentation in vivo has often been stressed $[20,22]$. From numerous studies, it is clear that extensive traffic and intimate contact of the cells in the spleen is necessary for the generation of the immune response. MZM degrade the entering antigens which are then transferred to secondary cells. It is not clear whether MMM behave differently from MZM in this respect. The strategic localization of MMM at the border of the marginal zone and close to the PALS (Fig. 4b) makes them ideal candidates for the transfer of antigens to antigen presenting $B$ cells or perhaps interdigitating dendritic cells in the inner PALS. However, it is not clear which factor determines the specific uptake of iscom by MMM while other antigens, i.e. proteins, erythrocytes, bacteria or RV-BPL, are not found in this cell population. $\mathrm{RV}$-iscom and empty iscom matrix localized in the same way. This finding demonstrates that the presence or absence of proteins in the iscom matrix does not influence localization behavior or the involvement of a particular subset of macrophages. Since low amounts of liposomes do not preferentially localize in MMM, a direct influence of cholesterol in this phenomenon can also be excluded, leaving Quil-A or the uncommon structure of the iscom as possible factors.

The labeled iscom we detected in the splenic follicles within hours after administration could be indicative for complement-mediated uptake [18] and a possible role for follicu- 
lar-dendritic cells in antigen presentation. As reviewed by Kosco [23] follicular dendritic cells have the capacity to pass antigen to B cells which in turn are capable of processing and presenting it to $T$ cells, leading to germinal center formation [24]. On the other hand, macrophages can also efficiently present antigen to $\mathrm{T}$ cells, thereby functionally implicating subsets such as the MMM until proven otherwise, as it the case for the suppressive MZM. Notwithstanding the follicular localization of the iscoms, a role for follicular-dendritic or -B cells in the presentation and processing of iscom, which is also partly major histocompatibility complex class I-dependent, remains to be proven.

Until now, only one study has described the early events in the immune response following i.p. injections of iscoms compared to micelles. In this study, it was shown that there was a significant increase in the inflammatory response and an increase in the uptake of radiolabeled antigen in the spleen following immunization with iscom [7]. Even more interesting was the observation that a larger amount of antigen in iscom is retained in the spleen when compared to antigens in micelles. In the present study, fluorescence in spleen and lymph nodes could still be demonstrated after 1 week, but not differences were observed between RVBPL or iscom.

In conclusion, we have shown that macrophages from the marginal zone, and in particular the MMM, are involved in uptake and, therefore, probably also in processing and presentation of iscom-associated antigens. No evidence was found for direct interactions between iscom and $\mathrm{T}$ cells (iscom were not found in the main $\mathrm{T}$ cell areas). However, the follicular localization of iscom indicates direct activation of $\mathbf{B}$ cells, a not-unlikely mechanism in view of the fact that no differential macrophage localization was observed in the lymph nodes after s.c. or i.m. immunizations. Further studies, involving splenic macrophage elimination [25] to establish the role of MMM and MZM in the immune response against iscoms and viral antigens, are now underway.

We would like to thank Marc Schellekens and Nel Kors for excellent technical assistance, J. S. Teppema for electron microscopy, Dr. G. Kraal for monoclonal antibodies, Drs. G. van Steenis and W. Boersma for constructive criticism and Dr. N. van Rooijen for histological interpretation of the initial experiment.

Received December 20, 1994; accepted February 2, 1995.

\section{References}

1 Morein, B., Sundquist, B., Hoglund, S., Dalsgaard, K. and Osterhaus, A., Nature 1984. 308: 457.

2 Claassen, I. J. T. M. and Osterhaus, A. D. M. E., Res. Immunol. 1992. 143: 531.

3 Harris, J. R., Subcell. Biochem. 1989. 15: 39.

4 Binnendijk van, R. S., Baalen van, C. A., Poelen, M. C. M., de Vries, P., Boes, J., Cerundolo, V., Osterhaus, A. D. M. E. and UytdeHaag, F. G. C. M., J. Exp. Med. 1992. 176: 119.

5 Mowat, A. M., Donachie, A. M., Reid, G. and Jarrett, O., Immunology 1991. 72: 317.

6 Takahashi, H., Takeshita, T., Morein, B., Putney, S. and Germain, R., Nature 1990. 344: 873.

7 Watson, D. L., Lovgren, K., Watson, N. A., Fossum, B., Morein, B. and Hoglund, S., Inflammation 1989. 13: 641.

8 van Wezel, A. L., van Steenis, B., Hannik, C. A. and Cohen, H., Dev. Biol. Stand. 1978. 41: 159.

9 Fekadu, M., Shaddock, J. H., Ekstrom, J., Osterhaus, A., Sanderlin, D. W., Sundquist, B. and Morein, B., Vaccine 1992. 10: 192.

10 Thraenhart, O., Koprowski, H., Bögel, K. and Sureau, P. (Eds.), Progress in Rabies Control, Staples Printers, Rochester 1990 , p. 69.

11 Zalan, E., Wilson, C. and Pukitis, D., J. Biol. Stand. 1979. 7: 213.

12 Bunschoten, H., Dietzschold, B., Claassen, I. J. T. M., Klapmuts, R., UytdeHaag, F. G. C. M. and Osterhaus, A. D. M. E., Viral. Immunol. 1990. 3: 41.

13 World Health Organisation, Laboratory techniques in rabies, 3rd Edn., WHO, Geneva 1973, p. 279.

14 Claassen, E., Res. Immunol. 1992. 142: 235.

15 Brandtzaeg, P., McGhee, J., Mestecky, J., Sterzl, J. and Tlaskalova, M. (Eds.), Adv. Exp. Med. Biol. 1995, in press.

16 Kraal, G., Janse, M. and Claassen, E., Immunol. Lett. 1988. 17: 139 .

17 Claassen, E., Ott, A., Boersma,W., Deen, C., Schellekens, M., Dijkstra, C., Kors, N. and van Rooijen, N., Clin. Exp. Immunol. 1989. 77: 445.

18 Van den Eertwegh, A., Laman, J., Schellekens, M., Boersma, W. and Claassen, E., Eur. J. Immunol. 1992. 22: 719.

19 Claassen, E., Res. Immunol. 1991. 142: 315.

20 Van den Eertwegh, A., Boersma, W. J. A. and Claassen, E., Crit. Rev. Immunal. 1992. 11: 337.

21 Imhof, B., Berrih-Aknin, S. and Ezine, S. (Eds.), Lymphatic tissues and in vivo immune responses, Marcel Dekker, New York 1991, p. 855.

22 Van Rooijen, N., Immunol. Today 1990. 12: 436.

23 Kosco, M. H., Curr. Opin. Immunol. 1991. 3: 336.

24 Gray, D., Kosco, M. H. and Stockinger, B., Int. Immunol. 1991. 3: 141.

25 Van Rooijen, N., J. Immunol. Methods 1989. 124: 1. 